\title{
Pediatría basada en la Evidencia
}

Publicado en Internet: 25-septiembre-2014

Álvaro Gimeno Díaz de Atauri: agimenodatauri@gmail.com

\author{
A. Gimeno Díaz de Atauri ${ }^{a}$, V. Modesto i Alapont ${ }^{b}$ \\ aServicio de Pediatría. Hospital Universitario Puerta de Hierro-Majadahonda. Madrid. España \\ - bUCIP. Hospital Universitari i Politècnic La Fe. València. España.
}

Se ha publicado recientemente un artículo sobre el impacto de las distintas estrategias de vacunación en España ${ }^{1}$. Los autores concluyen que en España las infecciones graves por varicela han disminuido desde la puesta en marcha del programa de vacunación rutinaria en 2006. Esta disminución ha sido significativamente mayor en las comunidades autónomas que se decidieron por la estrategia de vacunar con dos dosis a todos los niños de 15-18 meses, frente a las que optaron por vacunar solo a los adolescentes susceptibles. Esta diferente estrategia no ha afectado a la tasa de hospitalización por herpes zóster, que ha ido incrementándose ligeramente, principalmente en los mayores de 84 años.

Palabras clave:

Tras la lectura crítica de dicho artículo, se puede concluir que, efectivamente, la incidencia de varicela

- Varicela grave se reduce con la vacunación de los adolescentes (de 10 a 14 años) sanos susceptibles y que este beneficio se duplica con la estrategia de vacunar a todos los niños de 15-18 meses de edad. Aunque no queda demostrada una relación causal con la vacunación, la incidencia global de herpes zóster grave sigue una tendencia de incremento progresivo, sobre todo en los ancianos. Por este motivo, la eficien-

- Hospitalización

- Herpes zóster

- España

- Epidemiología

\section{The varicella vaccination programme decreases the rate of pediatric severe varicella, but it does not change herpes zoster related hospitalizations}

In a recently published study ob the impact of different varicella vaccination strategies in Spain ${ }^{1}$, the authors conclude that severe varicella infections have decreased since routine varicella vaccination in Spain (2006). This decrease was significantly higher in regions including the vaccine at 15-18 months of age compared with those vaccinating only susceptible adolescents. The hospitalization rate related to herpes zoster slightly increased (mainly in the $>84$ age group). No significant differences were found

Key words:

- Varicella

- Vaccines

- Hospitalization

- Herpes zoster - Spain

- Epidemiology in herpes zoster hospitalization rates regarding the varicella vaccination strategies among regions.

After the critical appraisal of this study, it can be said that the incidence of severe varicella is actually reduced with varicella vaccination of susceptible adolescents. This benefit doubles vaccinating children at 15-18 months of age. The incidence of severe zoster is steadily growing, especially in the elderly. Although there is no evidence of a causal link with the vaccine, this issue is essential to establish the varicella vaccination social cost-effectiveness.

Este artículo se publica simultáneamente con la revista electrónica Evidencias en Pediatría (www.evidenciasenpediatria.es).

Cómo citar este artículo: Gimeno Díaz de Atauri A, Modesto i Alapont V. La vacunación rutinaria del virus varicela-zóster reduce la incidencia de varicela grave, sin alterar las hospitalizaciones por herpes zóster. Rev Pediatr Aten Primaria. 2014;16:247-50. 


\section{REFERENCIA BIBLIOGRÁFICA}

Gil-Prieto R, Walter S, González-Escalada A, GarcíaGarcía L, Marín-García P, Gil-de-Miguel A. Different vaccination strategies in Spain and its impact on severe varicella and zoster. Vaccine. 2014;32: 277-83.

\section{RESUMEN ESTRUCTURADO}

Objetivo: describir la evolución de las formas graves de infección por el virus varicela-zóster (VVZ) entre 2005 y 2010 tras el inicio de la vacunación sistemática en España en 2006. Evaluar posibles diferencias entre vacunar solo a los adolescentes sanos susceptibles (vacunación de adolescentes [VA]) o a todos los lactantes de 15-18 meses (vacunación de lactantes [VL]).

Diseño: estudio de cohortes poblacional retrospectivo.

Emplazamiento: hospitales públicos españoles recogidos en el Conjunto Mínimo Básico de Datos (CMBD) del Ministerio de Sanidad.

Población de estudio: población española.

Evaluación de la intervención: para comparar las dos estrategias de vacunación (VLy VA) se consideraron por separado las poblaciones de las cuatro comunidades autónomas (CC. AA.) en las que se estableció VL (Madrid, Navarra, Ceuta y Melilla) y las del resto de CC. AA., que instauraron VA. Se incluyeron también en el análisis los datos referentes a los años anteriores a la comercialización de la vacuna (publicados en un estudio previo).

Medición del resultado: se ha medido la incidencia poblacional de varicela grave mediante la tasa anual de hospitalizaciones por este motivo (recuento anual de hospitalizaciones dividido por la población anual en riesgo). Los datos se han recopilado del CMBD. Se ha estratificado por edades: menores de cinco años, de cinco a nueve años y mayores de nueve años para ingresos por varicela; y menores de 50 años, de 50 a 54, de 55 a 59, de 60 a 64, de 65 a 69, de 70 a 74, de 75 a 79, de 80 a 84 y mayores de 84 años para los ingresos por herpes zóster (HZ). El análisis se ha realizado ajustando un modelo especial de regresión multivariable de Poisson, Ilamado de diferencias en las diferencias, que modeliza las diferencias entre dos o más cohortes (por ejemplo, grupo de tratamiento y grupo control) en diferentes periodos de tiempo. La variable de resultado es la tasa (densidad de incidencia) en cada comunidad autónoma para cada año del seguimiento, y las variables explicativas son tres: una variable binaria que identifica a las CC. AA. que se decidieron por $\mathrm{VL}$, para evaluar su efecto frente a VA; una variable binaria que separa la serie en dos periodos, el previo y el posterior a 2006 (fecha de inicio de la vacunación), e identifica el efecto que induce la decisión de vacunar frente a no vacunar; y la variable de interacción de las otras dos. Las tres variables explicativas resultaron significativas para el modelo. Todas las tasas, para cada grupo etario, se expresan por 100000 habitantes-año.

Resultados principales: la decisión de vacunar disminuye la tasa anual de incidencia de hospitalizaciones por varicela, que cayó significativamente $(p=0,036)$ en toda la población española, desde 4,53 en 2005 hasta 2,95 en 2010. El efecto principal se observa en los niños menores de cinco años: desde 46,77 (intervalo de confianza del 95\% [IC 95\%]: 43,81 a 49,73) a 26,55 (IC 95\%: 24,50 a 28,60). Comparado con VA, VL reduce en seis años a la mitad la densidad de incidencia de varicela grave (se multiplica por un factor de 0,54 [IC 95\%: 0,39 a 0,77]). Así, en las 15 CC. AA. que adoptaron VA, la tasa se redujo un 37\%, desde 5,12 (IC 95\%: 4,87 a 5,35) hasta 3,22 (IC 95\%: 3,04 a 3,40). En las cuatro CC. AA. que se decidieron por la VL, cayó un $78 \%$, de 6,77 (IC 95\%: 6,14 a 7,40) a 1,46 (IC 95\%: 1,18 a 1,74). Este efecto se vio principalmente en menores de cinco años.

No se constató que la decisión de vacunar ni el tipo de estrategia vacunal influyeran en la tasa anual de incidencia de hospitalizaciones por $\mathrm{HZ}$ $(p=0,794)$, que se mantuvo constante para toda la población durante los seis años del estudio alrededor de 10,33. En el grupo de los mayores de 84 años sí hubo un incremento significativo, desde 
53,73 (IC 95\%: 51,94 a 55,52) antes de 2006 hasta 97,68 (IC 95\%: 91,62 a 103,73) en 2010.

Conclusión: la vacunación sistemática de varicela de los adolescentes (de 10 a 14 años) sanos susceptibles, reduce en los seis años estudiados la incidencia de varicela grave. Esté efecto beneficioso se duplica en caso de vacunar además a todos los lactantes de 15-18 meses. El efecto principal se observa en niños menores de cinco años. La incidencia de $\mathrm{HZ}$ grave se mantiene estable en la población general pero sigue una importante tendencia ascendente en los mayores de 84 años.

Conflicto de intereses: no declarados.

Fuente de financiación: Fondo de Investigaciones Sanitarias. Uno de los autores ha sido becado parcialmente por la Fundación Caja Madrid.

\section{COMENTARIO CRÍTICO}

Justificación: la vacuna para varicela en sujetos sanos ha demostrado disminuir las hospitalizaciones y los fallecimientos en relación con el VVZ. El Comité Asesor de Vacunas de la Asociación Española de Pediatría (CAV-AEP) recomienda la administración universal en dos dosis, a los 12 y a los 24 meses de edad $^{2}$. En España existen 19 calendarios vacunales distintos. En la propuesta de unificación del Ministerio de Sanidad, Servicios Sociales e Igualdad para 2014 se opta por vacunar a los 12 años de edad solo a los niños que no hayan pasado la enfermedad. Este estudio es enormemente pertinente, pues analiza el impacto de las dos estrategias vacunales en España.

Validez o rigor científico: la pregunta de investigación está claramente planteada. La medición de la exposición y su efecto parece independiente y ciega. Hay una adecuada precedencia temporal de la vacunación sistemática sobre las hospitalizaciones y hay un tiempo de seguimiento suficiente para que el efecto aparezca. No se registra la cobertura vacunal anual en las distintas CC. AA. ni se incluye este dato en el modelo estadístico, tampoco las tasas globales de hospitalización por cualquier causa. Podría tener importancia conocer, en las CC. AA. con estrategia VA, la tasa de vacunación a lactantes autofinanciada por las familias. La variable hospitalización es adecuada para evaluar enfermedad grave por VVZ, aunque sería interesante tener también datos sobre neuralgia posherpética, patología muy invalidante y duradera.

Podrían plantearse problemas de representatividad en la muestra. No se especifica de dónde se han obtenido los datos de las poblaciones anuales de las diferentes CC. AA. (denominador de las tasas). En un artículo previo del mismo grupo sobre el mismo tema ${ }^{3}$ se obtuvieron de las proyecciones del censo de 1998-2004 ajustadas al 97,7\% de la población cubierta por los hospitales incluidos en la base de datos del CMBD, por lo que es probable que esa sea la fuente de estos datos. También se desconoce si la distribución por edades de la población registrada en el CMBD es idéntica a la de la población del censo y entre las distintas CC. AA. Se desconocen las pérdidas por casos mal codificados o ingresados en centros privados (hasta un tercio de las camas hospitalarias en España ${ }^{4}$. El manejo de la confusión se ha realizado adecuadamente mediante el modelo multivariable de regresión de Poisson. Sin embargo, no se dan datos sobre el grado de sobredispersión del modelo, factor fundamental para la validez. A pesar de todo, las conclusiones del trabajo parecen robustas. El efecto de la vacuna es muy importante y, además, la investigación se ha realizado aprovechando una coyuntura excepcional en nuestro país.

Importancia clínica: el efecto de la vacuna sobre la hospitalización por varicela parece muy relevante y es mucho mayor con $V L$ que con VA. Los datos son congruentes con la bibliografía internacional sobre el tema. En un estudio en Canadá ${ }^{5}$, cuyo calendario incluye la vacuna universal a los 12-15 meses, también se encontró un descenso importante en los ingresos por varicela, especialmente en los menores de cinco años (83\%).

La reexposición continuada al VVZ circulante, al reactivar la respuesta inmunitaria, podría proteger a los ancianos del $\mathrm{HZ}$ (hipótesis del exógeno). Por ello es posible que la vacunación universal aumente la incidencia del $\mathrm{HZ}$ en ancianos. Una revisión 
sistemática reciente concluye que este fenómeno existe, aunque es muy heterogéneo y difícilmente cuantificable 6 . En el presente estudio se objetivó un aumento muy significativo (casi del doble) de ingresos por $\mathrm{HZ}$ en mayores de 84 años, aunque la relación de causalidad con la vacuna no se ha podido acreditar, entre otros motivos, porque en ese grupo de edad aumentan las hospitalizaciones por todas las causas. En EE. UU. se ha observado un aumento progresivo de incidencia de $\mathrm{HZ}$ en mayores de 65 años desde antes de la introducción de la vacuna en el calendario, sin que la vacunación universal supusiera un punto de inflexión en esa tendencia ${ }^{7}$. En este estudio no se analizan costes. En otros, la vacuna solo parece coste-efectiva si se asume que no se asocia a un aumento del $\mathrm{HZ}^{8}$.

\section{BIBLIOGRAFÍA}

1. Gil-Prieto R, Walter S, González-Escalada A, GarcíaGarcía L, Marín-García P, Gil-de-Miguel A. Different vaccination strategies in Spain and its impact on severe varicella and zoster. Vaccine. 2014;32:277-83.

2. Moreno-Pérez D, Álvarez García FJ, Arístegui Fernández J, Cilleruelo Ortega MJ, Corretger Rauet JM, García Sánchez N, et al.; en representación del Comité Asesor de Vacunas de la Asociación Española de Pediatría. Calendario de vacunaciones de la Asociación Española de Pediatría: recomendaciones 2014. An Pediatr (Barc). 2014;80:55.e1-55.e37.

3. Gil A, Gil R, Álvaro A, San Martín M, González A. Burden of herpes zoster requiring hospitalization in Spain during a seven-year period (1998-2004). BMC Infect Dis. 2009;9:55.

4. Catálogo Nacional de Hospitales 2011. Actualizado a 31 de diciembre de 2010. Ministerio de Sanidad, Po-
Aplicabilidad en la práctica clínica: la estrategia VL parece efectiva. Debería plantearse una evaluación rigurosa de la eficiencia en España y su influencia sobre la equidad del sistema sanitario español, antes de proceder a la toma de decisiones.

\section{CONFLICTO DE INTERESES}

Los autores declaran no presentar conflictos de intereses en relación con la preparación y publicación de este artículo.

\section{ABREVIATURAS}

CAV-AEP: Comité Asesor de Vacunas de la Asociación Española de Pediatría - CC. AA.: comunidades autónomas - CMBD: Conjunto Mínimo Básico de Datos • HZ: herpes zóster • IC 95\%: intervalo de confianza del 95\% • VA: vacunación de adolescentes $\bullet$ VL: vacunación de lactantes $\bullet$ VVZ: virus varicela-zóster.

lítica Social e Igualdad [en línea]. Disponible en http://publicacionesoficiales.boe.es/detail.php?id= 030086011-0001

5. Waye A, Jacobs P, Tan B. The impact of the universal infant varicella immunization strategy on Canadian varicella-related hospitalization rates. Vaccine. 2013; 31:4744-8.

6. Ogunjimi B, Van Damme P, Beutels P. Herpes Zoster Risk Reduction through Exposure to Chickenpox Patients: A Systematic Multidisciplinary Review. PLoS One. 2013;8:e66485.

7. Hales CM, Harpaz R, Joesoef MR, Bialek SR. Examination of links between herpes zoster incidence and childhood varicella vaccination. Ann Intern Med. 2013;159:739-45.

8. Bilcke J, van Hoek AJ, Beutels P. Childhood varicellazoster virus vaccination in Belgium: cost-effective only in the long run or without exogenous boosting? Hum Vaccin Immunother. 2013;9:812-22. 